\title{
Propuesta metodológica para estudiar los costes de reactivación de un espacio de montaña
}

\author{
M. ${ }^{a}$ Teresa Rubio Benito
}

\section{INTRODUCCIÓN}

La delimitación de las zonas de montaña es un proceso de suma transcendencia ya que, según cómo se realice y los criterios que se utilicen para su clasificación, las consecuencias serán diferentes de cara a la aplicación de las medidas políticas que para ella se establezcan, máxime si tenemos en cuenta que unos criterios de actuación únicos pueden repercutir de distinta manera dadas las singularidades territoriales y socio-económicas de estas zonas. Los efectos pueden ser negativos bien por una excesiva amplitud o por restricciones demasiado rígidas a la hora de considerar los baremos de delimitación (Fluvia Font, 1982).

La gestión de la montaña ha de realizarse en base a una política diseñada para la misma (Abreu y Pidal, 1981) pero, dentro de la amplitud que esta gestión permite, hemos de adaptarnos a los criterios administrativos establecidos a nivel nacional o regional, que a su vez están íntimamente relacionados o coordinados con los respectivos a nivel supranacional, dentro del marco en el que se aplican en la CEE. No obstante, hemos de tener en cuenta que si la política comunitaria no es asumible en su totalidad en todos los paises mucho menos lo es a nivel de región y de difícil aplicación en el ámbito comarcal.

* Dpto. de Geografía. Universidad Nacional de Educación a Distancia. 


\section{CRITERIOS UTILIZADOS EN LA DELIMITACIÓN DE ÁREAS DE MONTAÑA EN ESPAÑA}

Conscientes de las limitaciones estructurales que presenta cada territorio y las dificultades que supone aplicar unos baremos estandarizados para todas las comarcas, se desarrolla en el seno de la CEE una política sectorial que tiende a aplicar medidas concretas en las zonas con problemas socioestructurales especificos, que les impiden salir o superar los umbrales mínimos de depresión y alcanzar un estatus similar al resto de las zonas consideradas social y económicamente viables y progresivas (Elena Roselló, 1985).

Para establecer unos baremos adaptables a las zonas de montaña, los distintos paises han fijado sus criterios de delimitación, utilizando como parámetros prioritarios la pendiente y la altitud, por la influencia negativa que éstos pueden tener en el desarrollo de las actividades humanas. Estos parámetros se complementan con otros indicadores referidos al desequilibrio demográfico a nivel estructural y espacial, a la producción final agrícola, etc. En España si aplicamos estos mismos criterios de altitud (600 m. en Francia, Italia, 800 en Alemania, Grecia), el 56,02 por 100 del territorio está por encima de los $600 \mathrm{~m}$. y a más de $800 \mathrm{~m}$. el $35,37 \%$, aumentando el porcentaje al $72,56 \%$ si consideramos los $400 \mathrm{~m}$.

La delimitación se ha llevado a cabo en nuestro país por una Comisión creada al respecto, por Real Decreto 2717/1983, de 5 de octubre, en cumplimiento del art. 24 de la Ley de Agricultura de Montaña (LAM). Los criterios que se han tenido en cuenta son los siguientes:

- El $80 \%$ de la superficie del término municipal debe encontrarse por encima de la cota de los $1.000 \mathrm{~m}$.

- El término municipal debe tener una pendiente media igual o superior al $20 \%$.

También se pueden considerar como zonas de montaña aquellas que, teniendo vocación agraria y no alcanzando los umbrales mencionados, éstos influyan o provoquen limitaciones para el desarrollo de la agricultura. El Real Decreto $2164 / 84$, de 31 de octubre, las califica como equiparables y con el fin de adecuar su delimitación con los criterios comunitarios el Real Decreto 1083/1986, de 30 de mayo, considera como tales aquellas zonas que combinen una situación mixta de pendiente media igual o superior al $15 \%$ y la situación de su término municipal se 
encuentre, en cotas superiores a los $600 \mathrm{~m}$, en el $80 \%$ del mismo. Tanto en un caso como en otro el establecimiento de los Programas de Ordenación y Promoción es el mismo.

\section{MEDIDAS DE ACTUACIÓN QUE PERMITEN LAS LEYES}

Para resolver estos problemas que frenan o impiden el desarrollo de las zonas consideradas de montaña, las actuaciones específicas de cada territorio se adecúan a la política socioestructural comunitaria, de acuerdo con la Directriz 268/75, «sobre agricultura de montaña y de ciertas zonas desfavorecidas". Con ello se pretende dar un paso importante en materia socioestructural, pasando de aplicar medidas "horizontales" y uniformes a una política basada en medidas específicas para territorios específicos (Gómez Benito, y otros, 1987), atendiendo a los aspectos sociales y medioambientales además de los exclusivamente productivistas.

Estas medidas se complementan con las propuestas en el Reglamento (CEE) 797/85, del Consejo de 12 de marzo, sobre «mejora de la eficacia de las estructuras agrarias" que hace referencia a las zonas de montaña en lo que respecta a determinadas ayudas y exenciones que deben tener las mismas. Además propone una mayor integración intersectorial al remarcar los objetivos que deben perseguirse en el sector agrario, industrial y de servicios, acompañadas por una mejora en la; infraestructura. En definitiva, este Reglamento resume las exigencias apreciadas por la experiencia de más de una década de política socioestructural, remarcando que el desarrollo de las zonas de montaña ha de ser integral, basado en criterios comunitarios y coordinando tanto acciones como financiación procedentes de diversos Organismos y Entidades públicos o privados (DGA, 1985).

Por su parte la Constitución española, en su artículo 130.2, exige de las Administraciones Públicas que dediquen un tratamiento especial a las zonas de montaña, sobrepasando el estricto ámbito agrícola e intentando compatibilizar las ayudas externas con un cambio de mentalidad, que propicie actuaciones imaginativas, para que las inversiones sean económicamente rentables.

La Ley 25/1982, de 30 de junio (LAM), reconoce la singularidad de las zonas de montaña atendiendo a la demografía, los recursos, el medioambiente $y$ en general todas las actuaciones que posibiliten un desa- 
rrollo social y económico, haciendo especial hincapié en los aspectos agrarios. Tanto la LAM, como el Real Decreto 2164/1984, de 31 de octubre, que desarrolla la Ley y las normas posteriores, conciben la política sobre zonas de agricultura de montaña como una acción común de todas las Administraciones Públicas implicadas (estatal, autonómica y local) e, incluso, de la población montañesa, a través de las Asociaciones de Montaña y otras entidades representativas (Gómez Benito y otros, 1987).

El instrumento básico de intervención en las zonas de agricultura de montaña son los Programas de Ordenación y Promoción de Recursos Agrarios de Montaña. De acuerdo con la filosofía que propugnan, han de cuantificar los objetivos específicos en la medida de lo posible, previa delimitación de las zonas, y procurar una serie de acciones de cara a conseguir la adecuada ordenación, uso, defensa y protección de las mismas; se ha de crear la infraestructura correspondiente para el buen desarrollo de la actividad sectorial, capacitando al personal para que adquiera profesionalidad y obtenga los mejores beneficios en las actividades que tengan potencialidad para relanzar el sector correspondiente. Igualmente se contempla la evaluación y exposición de un catálogo completo de ayudas para poder cuantificar los gastos de conservación, funcionamiento y nueva creación que se deriven de la aplicación de la Ley. La duración del Programa es limitada y a ello se comprometen, en acción concertada, las distintas Administraciones implicadas.

La existencia de estos Programas implica la consecución de mayores beneficios en las zonas donde existan; estas ayudas serán de carácter general o específicas e individuales, a través de la Indemnización Compensatoria o por medio de ayudas a inversiones colectivas.

La responsable directa de la ejecución de la política de montaña es la correspondiente Comunidad Autónoma, que tiene competencias en casi todos los campos de la misma. No obstante, en todas sus actuaciones o decisiones puede, si to desea, pedir el asesoramiento a nivel estatal o comunitario por los cauces reglamentarios establecidos a tal efecto. Las actuaciones se pueden realizar a través de la Comisión de Agricultura de Montaña, los Comités de Coordinación de Zona, apoyados por las asociaciones locales, sindicatos, Ayuntamientos, etc.

\section{PLANTEAMIENTO DE OBJETIVOS EN UN ESTUdIO DE MONTAÑA}

La tradicional falta de atención prestada por la Administración a las áreas deprimidas y de montaña a la hora de planificar y organizar el 
Propuesta para estudiar los costes de reactivación de un espacio...

desarrollo del territorio de forma equilibrada y coherente, hace que para acometer cualquier alternativa de reactivación económico-social de estas zonas sea necesario, como paso previo, obtener una perspectiva integral e integradora, abordada con rigor y con imaginación, siempre y cuando se tengan muy presentes las interdependencias que se establecen entre el paisaje y el desarrollo de las actividades agrarias, industrial y de servicios, cuyo tratamiento debe hacerse de forma pormenorizada contando con la obligatoriedad ineludible de incorporar la opinión directa de la población del territorio objeto de estudio, como auténticos destinatarios del desarrollo (Anglada, Balcells y otros, 1980).

La situación creada en estas áreas es de gran complejidad. Por ello, no deben generalizarse a priori las implicaciones territoriales del cambio producido en los espacios de montaña, simplificándolo con frecuencia a situaciones de abandono y envejecimiento. Realmente, a veces constituyen auténticas bolsas de subdesarrollo que mantienen salarios bajos y rentas bajas; de aquí que, aumentar los niveles de productividad a largo plazo en estas zonas, sería la manera de reducir su incidencia negativa en la economía regional (Council of Europa, 1978).

Desde nuestro punto de vista una adecuada actuación requiere, en primer lugar, un análisis en profundidad de los caracteres e interdependencias que afectan a estas zonas, evaluando los costes que limitan su desarrollo, de forma pormenorizada, mediante la utilización y correlación de variables demográficas y espaciales, sin hacer ninguna restricción previa en el número de las elegidas o en el de individuos que conforman el espacio considerado. Los objetivos deben ser, a nuestro juicio (Rubio Benito, y otros, 1987), concretos y abarcables y podríamos resumirlos en tres principales:

- Conocer las particularidades físicas, demográficas, económicas y sociales de cada uno de los municipios que integran la zona objeto de estudio.

- Evaluar el nivel de inversión pública realizado en los últimos años, por habitante/año.

- Cuantificar los costes de la reactivación económico-social de la comarca.

\section{Metodología}

Para lograr los objetivos propuestos, tomaremos como unidad de análisis espacial el municipio, asignando a cada uno de ellos un elevado 
número de variables agrupadas en demográficas, funcionales e indicadores sociales (calidad de vida, bienestar, equipamiento). Aspectos territoriales de interés especial son los usos del suelo, localización de equipamientos, flujos de personas, mercancias e información y desplazamientos en general.

Para establecer las correlaciones entre la estructura socioeconómica y las variables de inversión, aplicaremos los principios metodológicos de análisis factorial y regresión sobre componentes principales. En función de lo anterior, delimitaremos unas zonas homogéneas que respondan a una misma tipología, con el fin de fijar determinados umbrales de desarrollo que permitan evaluar los costes de reactivación en cada una de ellas.

El uso de un elevado número de variables asignadas a toda una serie de unidades espaciales requiere la utilización de unas herramientas de trabajo adecuadas que permitan el manejo de tan numeroso caudal de conocimientos empíricos de carácter estadístico, reduciéndolo a sus características esenciales. La solución radica en el uso de un tratamiento informático para establecer las relaciones existentes entre el conjunto de individuos y el de variables (Mallo, 1985) agrupadas en distintos paquetes correspondientes a los aspectos físicos, demográficos, agrícola-ganaderos, industriales, comerciales y de servicios que constituyen lo que denominamos variables técnicas y luego las respectivas de inversión en infraestructura y sectores de actividad.

La metodología de tratamiento propuesta en base al programa BMDP-4M (Análisis factorial) y BMDP-4R (Regresión sobre componentes principales) fue desarrollada por el Departamento de Biomatemáticas de la Universidad de California, Los Ángeles (Grupo Chadule, 1980). Cada paquete de variables ha sido sintetizado en las respectivas salidas de ordenador que nos aportan los siguientes datos:

- Estadística descriptiva del grupo de variables (media, desviación standar, coeficiente de variación, etc.).

- La matriz de correlación.

- La comunalidad de las variables entre sí y de ellas con los factores.

- Matriz de cargos factoriales (Factor Leasing) que expresa la correlación entre cada variable original y las nuevas variables. En base a ella, se interpretan los resultados atendiendo a los coeficientes de correlación que superan el 0,5 , en la matriz resultante de la rotación ortogonal de los factores, realizado sistemáticamente en 
este programa para resaltar la correlación de los factores con determinadas variables.

Así, hemos obtenido de forma implícita una descripción de la estructura de dependencia en un espacio de menor dimensión, sin que por ello hayamos perdido demasiada información respecto a las variables originales. Pero lo que nos interesa primordialmente es averiguar las relaciones que existen entre los municipios (individuos) y el conjunto de variables. Por ello, la salida gráfica del ordenador nos proporciona, por una parte, la representación de las variables en la base ortonormal de las componentes principales reducidas y la de los individuos, en la base ortonormal del subespacio RP. Con la primera representación obtenemos la contribución de variables a la configuración del espacio reducido, la correlación entre variables y la de éstas con los componentes principales. Si los municipios o los individuos están bien representados, el significado vendrá dado por su posición respecto a los factores. Este resultado es de vital interés porque habremos pasado del nivel estadístico, al espacial.

\section{Propuesta de trabajo}

Para llegar a una adecuada estimación de las magnitudes económicas, es indispensable evaluar exhaustivamente los datos que nos proporcionan las fuentes primarias y los informes y censos estadísticos facilitados por los respectivos organismos públicos, a nivel nacional, regional, provincial y local, complementados con los sondeos y entrevistas llevados a cabo en sucesivas visitas de campo (Daumas, 1976).

La consulta y exploración de las fuentes estadísticas se ha de llevar a cabo de forma selectiva, de acuerdo con los objetivos que se persiguen; así, de entre los factores físicos consideraremos tan sólo la pendiente y la altitud, determinantes en la delimitación de áreas de montaña dentro de la Ley de Agricultura de Montaña, por entender que la pendiente es un factor limitante de la mecanización agrícola y por estar la altitud relacionada con las variables climáticas (temperatura, pluviometría, régimen de heladas, etc.) que afectan a los distintos cultivos; tradicionalmente la actividad humana se ha adaptado a la dureza de unos elementos naturales que se presentan como factores limitativos, tanto en su topografía como en los aspectos bioclimáticos, dado que el $40 \%$ del territorio se sitúa por encima de los $1.000 \mathrm{~m}$ de altura. Las condiciones hostiles para el desarrollo de una agricultura avanzada permiten por el contrario la explotación forestal y ganadera (Montserrat Recoder, 1974), 
ya que la abundancia de precipitaciones, especialmente de carácter nival, favorecen la abundancia de pastos naturales. No obstante, el proceso de cambio que ha impuesto la economía de mercado ha causado un impacto en las sociedades de economía poco desarrollada, como las de montaña, difícil de superar teniendo en cuenta la debilidad de efectivos poblacionales, resultado de los fuertes contingentes de emigración extracomarcal que se vienen sucediendo desde la revolución industrial y que se traducen en una bajísima densidad de habitantes por $\mathrm{Km}^{2}$ y una población absolutamente envejecida con escasa capacidad de iniciativa (Rubio Benito, y Castelió Puig, 1986).

Por ello nos parece de vital importancia establecer la relación existente entre potencial demográfico y posibilidades de desarrollo de acuerdo con el planteamiento de reactivación socioeconómica que se persigue, perfilando las estructuras demográficas de cada municipio según el último censo publicado, distinguiendo sexo, edad, ocupación por ramas de actividad económica, población activa en los distintos sectores y posibilidades de reactivación según el grado de envejecimiento y de reemplazamiento de la población, reforzando y constatando este aspecto, mediante el vaciado del Padrón de habitantes de, al menos, los dos últimos decenios.

Valoraremos a continuación el nivel de infraestructura, equipamiento y servicios en que se encuentra la comarca según los planes aprobados a nivel nacional, regional y provincial para establecer el punto de partida de una posible reactivación socioeconómica de la zona.

Asimismo analizaremos la actual estructura agraria a partir de la cual puedan establecerse pautas de desarrollo que permitan una mejora de la productividad y producción agraria existente, mediante las inversiones precisas (Fernández Cancio, 1980). Teniendo en cuenta la baja densidad de población y el elevado índice de envejecimiento que suelen darse en la montaña, no se pretende hacer un estudio de evaluación de la potencialidad agraria de la comarca, a partir de variables técnicas, de suelo, clima, etc. por entender que los costes de tal objetivo serían desproporcionados.

Una vez vaciados y consultados los distintos censos y programas de desarrollo agrario para la zona, nos interesa completar la información en cuanto a ocupación de la población activa y a su peso específico en el sector industrial, comercial y servicios en la comarca, a fin de conocer las peculiaridades productivas de cada municipio, ya que para llegar a una adecuada estimación de las magnitudes económicas es indispensable un buen censo de empleo, verificando sobre el terreno los datos e 
información obtenidos. Deberá prescindirse, en el cómputo de empresas, de aquellas que tengan menos de cuatro empleados, considerando que las que consignan en el apartado de personal cifras por debajo de ese mínimo, son establecimientos netamente familiares registrados como autónomos y de escasa entidad (Rubio Benito, y otros, 1987).

La evaluación del número de licencias de comercio al por menor y al por mayor de los establecimientos presentes en la comarca, nos pondrá de manifiesto la estructura comercial de la misma. De otra parte debe conocerse el número de licencias existentes para distintos servicios de hosteleria, reparaciones, alimentación, etc. de modo que junto a las variables de rentas sectoriales, total y municipal disponible, quede definida la comarca desde un punto de vista socioeconómico.

Establecida una primera caracterización municipal y/o espacial y/o sectorial, estaremos ya en condiciones de fijar correlaciones con otro conjunto de variables que se refieren a inversiones de todo tipo efectuadas con cargo a los presupuestos de distintas administraciones públicas (nacional, regional y provincial) en la última década, de tal manera que las variables que definiremos a continuación, nos permitirán establecer una serie de correlaciones que facilitarán, sin duda, la caracterización de los distintos municipios a partir de una serie de factores, tales como:

- Las características del medio físico donde se desarrolla la actividad rural y agrícola, de enorme importancia para la comarca.

- La estructura de la población municipal.

- El nivel de equipamiento y servicios de que dispone cada municipio.

- La estructura productiva del sector agrario.

- La cuantificación y localización espacial de las actividades industriales, comerciales, de construcción y de servicios.

- Las rentas municipales que se obtienen como resultado de la agregación de todas las demás variables.

\section{INTERPRETACIÓN DE VARIABLES Y COMPONENTES PRINCIPALES}

Efectuado el análisis de componentes principales para cada paquete de variables, resultan, según el número de ellas, dos o tres factores que 


\section{VARIABLES FÍSICAS}

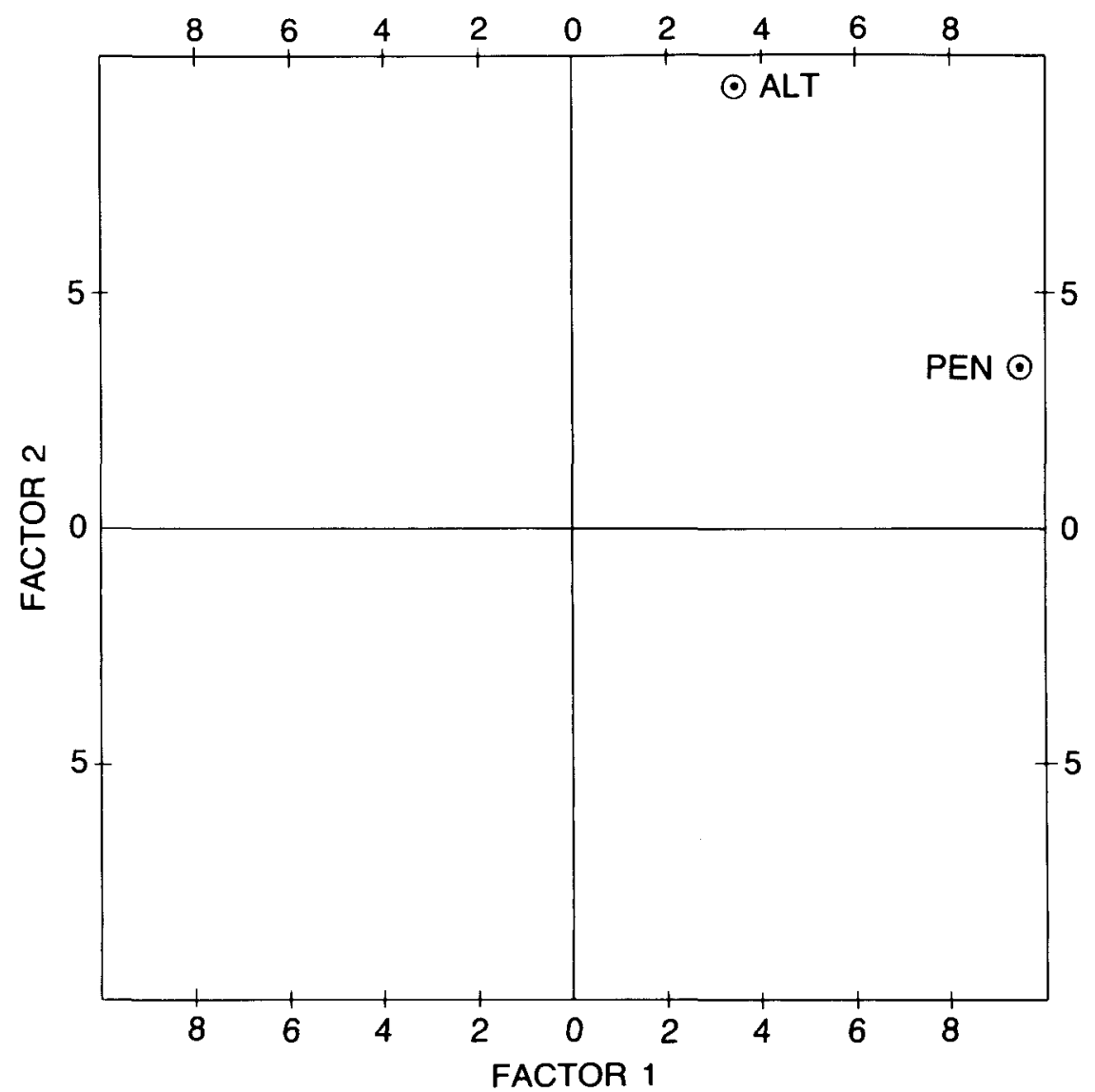

representan un tanto por ciento de la varianza explicada. Al observar el valor de cada variable en cada factor considerado, vemos que existen algunas altamente representadas, otras aceptablemente y algunas, con escasa o nula representación; si además en este último caso el factor tiene escasa aportación en la varianza, debemos prescindir del mismo, al objeto de concentrar la información sobre el paquete propuesto (físico, población, agricultura, etc.), en aquel o aquellos factores que contengan mayor significación. El valor de cada variable difiere según sea considerada en sí misma o respecto a la rotación, entre las demás, dentro del factor. 


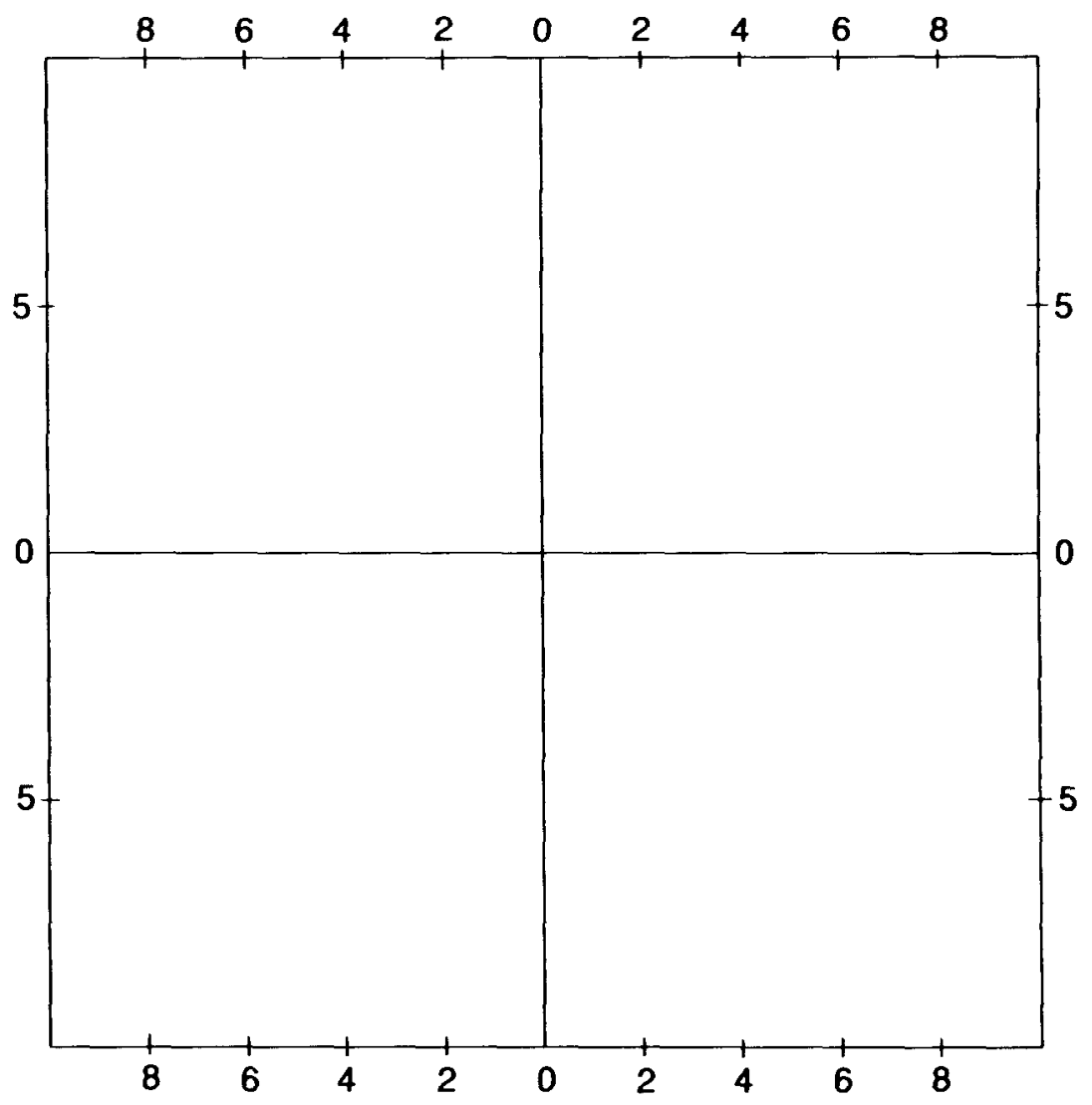

La representación de los municipios o de los individuos con relación al factor que se estudie puede dar también distintos grados de correlación. Es posible que, una vez analizados los resultados obtenidos del análisis de componentes principales, sea dificil establecer una zonificación coherente de acuerdo con el planteamiento inicial del trabajo, porque, en la montaña, la ausencia de homogeneidad es patente, dada la complicada orografia que tanto contribuye en la ruptura de la continuidad del espacio ya que una gran parte del territorio, se sitúa por encima de los $1.000 \mathrm{~m}$ de altura.

Si la mitad o más de los municipios presentan correlación negativa con todos los componentes, queda de manifiesto la desarticulación territorial existente en la mayor parte de la comarca, handicap importante a 


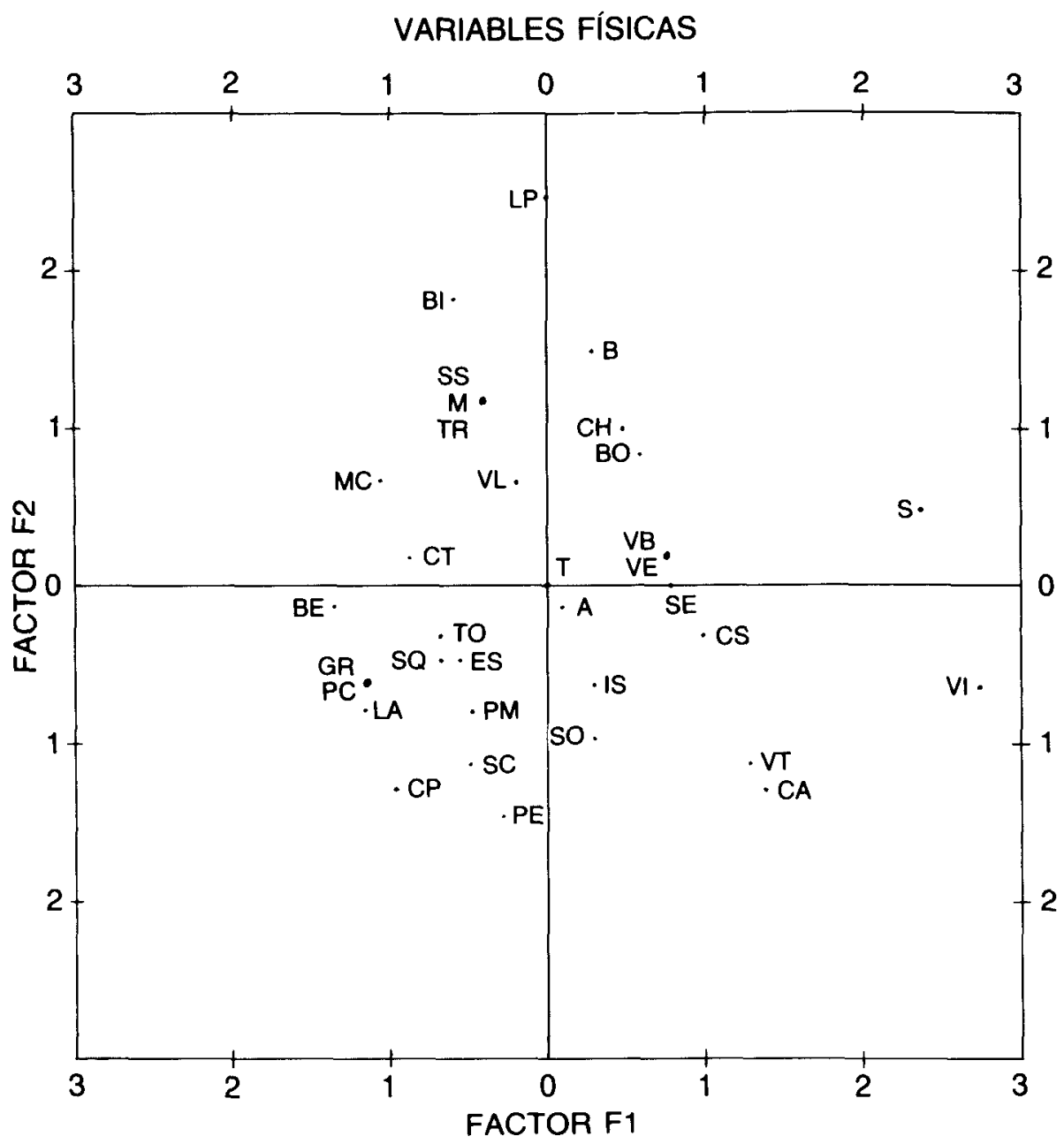

la hora de planificar cualquier programa de reactivación económica. Aquellos municipios que correlacionen muy positivamente y estén bien representados, serán sin duda los núcleos de cabecera sobre los que descansará el peso fundamental de la actividad económica.

A modo de ejemplo, citaremos el análisis de componentes principales (ACOPRI) realizado para las variables de carácter físico: pendiente (PEN) y altitud (ALT), que definen una comarca de alta montaña en el Pirineo oriental. Observando los resultados, vemos que, de los dos factores, el primero representa el $81,14 \%$ de la varianza explicada; ambas 
Propuesta para estudiar los costes de reactivación de un espacio...

variables son independientes, como pone de manifiesto el coeficiente de determinación, que resulta ser para cada variable del $38,79 \%$ y la rotación ortogonal efectuada, ya que en cada factor se explica el $94,40 \%$ de la varianza explicada por cada variable (ver cuadro 1), caracterizándose el factor $\mathrm{F} 1$ rotado por el valor pendiente y el F2 rotado por la altitud.

De las coordenadas de los individuos sobre el plano formado por los componentes principales con una calidad de representación del $100 \%$ resulta una primera clasificación de los núcleos de población. Así, al factor F2 los núcleos se ordenan del siguiente modo: Las Paúles, Bisaurri, Benasque, Sesué, Montanuy, Torre de la Rivera, Chía, Bonansa, Valle de Lierp, y al factor $F 1$ resultan altamente significativos Villanova y Sahún, que alcanzan pendientes del $50 \%$.

Asimismo, se aprecia una correlación positiva en los núcleos de Castejón de Sos, Veracruz, Valle de Bardaji y Seira, que a las variables físicas consideradas presentan una respuesta similar.

Hay que destacar que los municipios más sensibles al factor 2 se hallan ubicados en la cabecera y coinciden con los que mantienen una mayor extensión de superficie dedicada a prado y pastizal.

\section{CONCLUSIONES}

La aplicación metodológica del programa BMDP-4M (Análisis factorial) y BMDP-4R (Regresión sobre componentes principales) para estudiar los costes de reactivación de una comarca de alta montaña con 34 núcleos de población nos ha llevado (Rubio Benito y otros, 1987) a las siguientes conclusiones:

Que la correlación sobre CP indica, a partir de una serie de ecuaciones, la relación existente entre las variables estructurales (socioeconómicas) características del nivel de desarrollo de la comarca y las variables de inversión, lo que permite efectuar un seguimiento de la evolución de ambos tipos de parámetros, orientando el desarrollo en el sentido del menor coste y mayor eficacia. Todo ello porque entendemos que la regresión efectuada mide, de modo coyuntural, las relaciones desarrolloinversión en un momento dado. $Y$ entendida la reactivación como un proceso dinámico, al final de cada programa de inversión debe conocerse en torno a qué nuevas relaciones se efectúa el crecimiento económico. 
Siendo así, las nuevas inversiones han sido capaces de generar y potenciar la estructura económica y social de la comarca, dando como resultados nuevas y distintas demandas sociales, que se traducirán sin duda en un nuevo tipo de ecuaciones de regresión.

Que de la correlación no sólo surgen ecuaciones que vinculan variables socioeconómicas con otras de inversión, sino que además resultan una serie de ecuaciones que permiten conocer para un momento dado, el valor de una determinada inversión a partir de otras variables de inversión explicativas de la anterior, resultando asi una serie de parámetros que se constituyen en auténticos indicadores del valor estimado que alcanzará otro tipo de inversión.

Ni qué decir tiene que, conforme se vayan cumpliendo una serie de objetivos de inversión, serán nuevas las variables que cumplan tal objetivo director, todo ello como resultado del nuevo equilibrio que deberá alcanzarse, una vez satisfechas las demandas de infraestructura básica, de reestructuración agraria, etc.

Que es posible a partir de las actuales ecuaciones efectuar modelos de simulación, previa asignación de nuevas variables a partir de las existentes, estableciendo asi nuevos haces de ecuaciones de regresión, tantas como etapas de inversión se requieran. Todo ello puede permitir establecer un seguimiento del plan de reactivación a partir del modelo de desarrollo deseado, con el que puedan medirse los desajustes existentes entre tal plan y el resultado que dichas inversiones produzcan en la comarca objeto de reactivación. 


\section{BIBLIOGRAFÍA}

AdReu y PIDAL, J. M., 1981: "Condicionantes para la delimitación y gestión de las áreas de montaña españolas". Actas del Coloquio Hispano-Francés sobre Áreas de Montaña. Madrid, Ministerio de Agricultura.

ANGLADA, S.; BalCELls, E., y otros, 1980: “La vida rural en la montaña española (Orientaciones para su promoción)». Jaca. Monografía del Instituto de Estudios Pirenaicos, n. 107.

COUNCIL OF EUROPA, 1978: "La fonction des regions alpines dans l'amenegement du territoire européen». Strasbourg. Série d'Etudes, 20.

Daumas, M., 1976: La vie rurale dans le Haut Aragon Oriental. Madrid, CSIC.

D.G.A., 1985: Libro Blanco sobre las repercusiones en Aragón de la integración de España en la CEE. Zaragoza, DGA.

Elena Rosello, M., 1985: «Las zonas en depresión socioeconómica en la CEE». Rev. Estudios Agro-sociales, n. ${ }^{\circ}$ 132. Madrid, Ministerio de Agricultura.

Fernández Cancio, A., 1980: «Posibles aplicaciones de las técnicas de simulación a la investigación agraria». Madrid. INIA Serie: General, n. ${ }^{\circ} 6$.

Fluvia Font, M., 1982: “Notas sobre economía de montaña». Rev. Economia Industrial, n. ${ }^{\circ}$ 218, febrero. Madrid, Ministerio de Industria y Energía, p. 73-79.

Gómez Benito, C. y col., 1987: La política socio-estructural en zonas de agricultura de montaña en España y en la CEE. Madrid. SGT, Ministerio de Agricultura. 
Grupo Chadule, 1980: Iniciación a los métodos estadisticos en Geografia. Barcelona, Ariel.

Mallo, F., 1985: Análisis de componentes principales y técnicas factoriales relacionadas. Universidad de León.

MontSerRat ReCoder, P., 1974: "La utilización de recursos en relación con la estructura y estabilidad del ecosistema». Seminario sobre Estructura y Estabilidad del Ecosistema. Sevilla.

Rubio Benito, M. ${ }^{a}$ T. y Castello Puig, A. (dir.), 1986: Actas de las I Jornadas sobre Alternativas de Utilización del Espacio en Áreas de Montaña, febrero 1985. Zaragoza, Ministerio de Agricultura, DGA, CU de Huesca.

Rubio Benito, M. ${ }^{a}$ T. y otros, 1987: Costes de la reactivación económicosocial en áreas deprimidas y de montaña en Aragón. Madrid, Ministerio de Obras Públicas y Urbanismo (inédito). 